BULL. AUSTRAL. MATH. SOC.

MOS 2OM99, 22AI5

VOL. $4(197 \mid), 159-161$.

\title{
A universal semigroup
}

\section{Sidney A. Morris}

\begin{abstract}
J.H. Michael recently proved that there exists a metric semigroup $U$ such that every compact metric semigroup with property $P$ is topologically isomorphic to a subsemigroup of $U$; where a semigroup $S$ has property $P$ if and only if for each $x, y$ in $S, x \neq y$, there is a $z$ in $S$ such that $x z \neq y z$ or $z x \neq z y$.
\end{abstract}

A stronger result is proved here more simply. It is shown that for any set $A$ of metric semigroups there exists a metric semigroup $U$ such that each $S$ in $A$ is topologically isomorphic to a subsemigroup of $U$. In particular this is the case when $A$ is the class of all separable metric semigroups, or more particularly the class of all compact metric semigroups.

THEOREM. Let $A$ be any set of metric semigroups. Then there exists a metric semigroup $U$ such that for each $S$ in $A$ there is a mopping of $S$ into $U$ which is both a semigroup isomorphism and a homeomorphism.

Proof. Let $A=\left\{S_{i}: i \in I\right\}$. Without loss of generality, assume $\sup _{x, y \in S_{i}}\left[d_{i}(x, y)\right]=1$, for each $i$ in $I$, where $d_{i}$ is the metric in $S_{i}$. Adjoin an identity $e_{i}$ to each $S_{i}$ to give a semigroup $T_{i}$. Define a metric $d_{i}^{*}$ on $T_{i}$ as follows:

Received 23 September 1970. The author wishes to thank Dr Joseph Diestel for his useful comments. 


$$
d_{i}^{*}(x, y)= \begin{cases}d(x, y), & \text { if } x \text { and } y \in S_{i} ; \\ 1 & , \text { if } x=e_{i} \text { and } y \in S_{i} \\ 1 & , \text { if } x \in S_{i} \text { and } y=e_{i} \\ 0 & , \text { if } x=y=e_{i} .\end{cases}
$$

It can be verified easily that $d_{i}^{*}$ is a metric and furthermore this metric is compatible with the semigroup structure; that is each $T_{i}$ is a metric semigroup.

Let $U$ be the direct sum of $\left\{T_{i}: i \in I\right\}$. (See [1].) Define a metric $d$ on $U$ as follows:

Let $x$ and $y$ be any distinct elements of $U$. Then $x=\left\{x_{i}\right\}$ and $y=\left\{y_{i}\right\}$, where all but a finite number of $x_{i}$ and $y_{i}$ are equal to $e_{i}$. Put $d(x, y)$ equal to the sum of the non-zero $d_{i}^{*}\left(x_{i}, y_{i}\right)$, $i \in I$.

It is a routine matter. to check that $d$ is a metric and $U$ with this metric is a metric semigroup. Finally, note that $U$ does have the required properties.

COROLLARY. Let $A$ be the class of all metric separable semigroups or the class of all compact metric semigroups. Then there exists a metric semigroup $U$ such that for each $S$ in $U$, there is a mopping of $S$ into $U$ which is both a semigroup isomorphism and a homeomorphism.

Proof. We only have to verify that $A$ is a set. This follows immediately from the theorem of Urysohn ([2], p. 125) which states that every separable metric space is homeomorphic to a subset of the Hilbert cube.

\section{References}

[1] A.H. Clifford and G.B. Preston, The algebraic theory of semigroups, Vol. II (Math. Surveys 7 (II), Amer. Math. Soc., Providence, Rhode Island, 1967). 
[2] John L. Kelley, General topology (Van Nostrand, Toronto, New York, London, 1955).

[3] J.H. Michael, "A universal semigroup", J. Austral. Math. Soc. 11 (1970), 216-220.

University of Florida,

Gainesville,

Florida, USA. 